\title{
Assessment of seminal plasma free amino acids among fertile men
}

\section{Marwa O. Aboelwafa ${ }^{a}$,Tahia H.Seleem ${ }^{b}$, Hassan M. Ibraheem ${ }^{c}$, Mohammed H. Hassan}

${ }^{a}$ MedicalBiochemistry Department,Faculty of Medicine ,South Valley University,Qena,Egypt.

${ }^{\mathrm{b}}$ MedicalBiochemistry Department,Faculty of Medicine ,AssiutUniversity ,Assiut,Egypt.

${ }^{\mathrm{c}}$ Dermatology,Venereology,AndrologyDepartment,Qena University Hospital, SouthValleyUniversity,Qena,Egypt.

\section{Abstract:}

Background:Amino acids can elevate sperm count, motility, and morphology. Increasing the availability of these amino acids can help to improve male fertility certain foods can help to improve sperm parameters. This is due to the high concentration of specific amino acids. There are many amino acids involved in the production, development and maintenance of sperm.

Aims:assessment of seminal plasma free amino acids among fertile men.

Patients and methods: this study done on 50 fertile menassessment of free amino acids in their seminal plasma, Semen analyses were done and amino acids measured in seminal plasma using H.P.L C. Results:The median and range of their semen analysis parameters were :volume 2(3-3),liquefaction time 10(10-15), sperm concentration 60(50-75)million / $\mathrm{ml}$,total sperm motility80(80-80) \%, abnormal sperm35(30-40), viability 67.5(60-80)\%the range of seminal plasma leucine was(1.68-1.87), isoleucine (1.22-1.31),valine(0.33-0.53)tyrosine, (1.11-1.22),phenylalanine (0.79-0.89) ,histidine (1.28-1.51) ,Tryptophan (0.26-0.36).

Conclusions:Assessment of seminal plasma amino acids is very helpful in evaluation of male fertility.

Keywords: amino acids, fertility,seminalplasma,semen analysis.

\section{Introduction:}

Amino acids can elevate sperm count, motility, and morphology. Increasing the availability of these amino acids can help to improve male fertility certain foods can help to improve sperm parameters. This is due to the high concentration of specific amino acids. There are many amino acids involved in the production, development and maintenance of sperm, (Schachter et al., 1973)for example:arginine amino acid is found in high concentrations within sperm, particularly in sperm heads. It's also the precursor in the synthesis of spermidine, putrescine and spermine. These three compounds are all important components of sperm.
Although sperm may still be produced without adequate levels of arginine, Elevating Larginine availability will increase blood flow to the

reproductive organs. This makes it easier to develop and maintain erections. L-arginine is necessary for the production of nitric oxide, therefore indirectly facilitating healthy blood circulation and vessel dilation. Not only does this help to reduce any possible erectile dysfunction, it also improves nutrient supply to the developing 
sperm, supplement with L-arginine will boost sperm count. (Aydin et al.,1995).

The present study was carried out on 50 fertile men their age ranges from 24 to 35 years old.

The patients were selected from the outpatient clinics of dermatology,venereology and andrology department,Qena university hospitals in the duration from January and June 2018 to evaluate normal levels of different free amino acids in fertile men.

\section{Data collection:}

A- History suggestive of epididymo-orchitis, prostatitis, genital trauma, testicular torsion, cryptorchidism, inguinal or genital surgery

B- History of chronic medical diseases, or receiving hormonal therapy or chemotherapy or radiotherapy.

C- Genital diseases such as (cryptorchidism ,maldescended testes or any uro-genital anomalies, obstructive azoospermia ,urinary tract infection or varicocele,prostatitis,genital trauma and testicular torsion(

D- Hypertension, autoimmune or inflammatory disorders, coronary artery disease, diabetes mellitus, or chronic renal failure or those receiving hormonal therapy or chemotherapy were excluded from the study.

E- Abnormal hormonal profile and presence of any endocrinopathy.

\section{Ethical consideration:}

All patients will be included in the study after taking a written consent from the patient's parents after full explanation of the purpose, nature and risks of all procedures used according to the ethical committee of the Qena University hospital.

\section{Physical examination:}

A-General examination including:

\section{Patients and methods:}

1-Measurement of patientsweight, height, for BMI calculation and BP.

2-Assessment of gynecomastia and secondary sex characteristics such as hair distribution

\section{B-Genital examination:}

1-Examination of the penis: including the location of the urethral meatus.

2-Palpation of the testis and measurement of their size.

3-Presence and consistency of both vasa epididymis Presence of varicocele

4-Secondary sex characteristics including body habitus ,hair distribution, and breast development

5-Digital rectal exam.

C-Investigation:

\section{Semen analysis:}

Semen samples were collected from the subjects , after 3-5 days of sexual abstinence, by masturbation. Macrosopic and microscopic semen analysis was performed according to $\mathrm{WHO}$ criteria (WHO, 2010).

After allowing 30min for liquefaction, Then each semen sample was centrifuged at 4000rpm for 10 min and the supernatent clear seminal plasma was transferred into $1 \mathrm{ml}$ cryotubes and stored at - 80C till time of biochemical analysis of amino acids.

Amino acid assay: The assays were performed by using an automatic amino acid analyzer.

\section{Statistical analysis:}

Data were analyzed using IBM SPSS statistics for windows version 20(Armonk,NY:IBM Corp).The data were tested for normalityusingKolmogrovSmirnov and Shapiro-Wilktests. Qualitative data were presented as number, percentage,Quantative 
data were expressed as mean and standard deviation( if were parametric data),median and interquartile range (for non parametric data).

\section{Results:}

The study has been conducted on 50 fertile men with their age ranges from 24 to 35 years old.

The median and range of their semen analysis parameters were :volume 2(3-3), liquefaction time 10(10-15), sperm concentration 60(50-75)million / $\mathrm{ml}$,total sperm motility80(80-80) \%, abnormal sperm35(30-40), viability 67.5(60-80)\%

The median and range of essential amino acids in seminal plasma were:valine, 0.42, (0.33-0.53), isoleucine1.29, (1.22-1.31), leucine 1.78 , (1.681.87) Phenylalanine $0.83(0.79-0.89)$ Histidine, 1.5 (1.28-1.51) Tryptophan,0.33(0.26-0.36), Table 1

The median and range of basic amino acids was :ornithine ,2(1.7-2.3) Lysine, 2.6 (2.1-2.8), Arginine , 1.44( 1.39-1.59).the median and range of acidic amino acid aspartic acid1.2,(1-1.5) and of hydroxy amino acids threonine1.1 (1.081.2)tyrosine, 1.14 (1.11-1.22), Table 2.

Table 1. Seminal plasma levels of essential amino acids

\begin{tabular}{|l|l|}
\hline Semen plasma amino acids & Fertile men \\
$\begin{array}{l}\text { Mmol/ mg seminal plasma } \\
\text { protein })\end{array}$ & $(\mathrm{N}=50)$ \\
\hline Valine & $0.42(0.33-0.53)$ \\
\hline Isoleucine & $1.29(1.22-1.31)$ \\
\hline Leucine & $1.78(1.68-1.87)$ \\
\hline Phenylalanine & $0.83(0.79-0.89)$ \\
\hline Histidine & $1.5(1.28-1.51)$ \\
\hline Tryptophan & $0.33(0.26-0.36)$ \\
\hline
\end{tabular}

\section{Table2:seminal plasma levels of basic, acidic and hydroxyamino acids:}

\begin{tabular}{|l|l|}
\hline $\begin{array}{l}\text { Semen plasma amino acids } \\
(\mu \mathrm{mol} / \mathrm{mg} \text { seminal } \\
\text { plasma protein) }\end{array}$ & \begin{tabular}{l}
$(\mathrm{N}=50)$ \\
\hline Ornithine
\end{tabular} \\
\hline Lysine & $2(1.7-2.3)$ \\
\hline Arginine & $2.6(2.1-2.8)$ \\
\hline Aspartic acid & $1.44(1.39-1.59)$ \\
\hline Tyrosine & $1.2(1-1.5)$ \\
\hline Threonine & $1.14(1.11-1.22)$ \\
\hline
\end{tabular}

\section{Discussion:}

Amino acids play important role in male fertility , Little is known about the significance of free amino acids in semen (Silvestroniet al.,2009) so this study was carried on 50 fertile men to evaluate levels of different aminoacids in seminal plasma among fertile men and found that the range of: leucine(1.68-1.87), valine (0.33-0.53), isoleucine (1.22-1.31) ,table 1 regarding branched chain amino acids , and the range of tyrosine(1.111.22), phenylalanine (0.79-0.89), histidine (1.281.51),tryptophan(0.26-0.36) ,table 2 regarding aromatic amino acids, the range of threonine (1.08-1.2),lysine (2.1-2.8), arginine (1.39-1.59)( table 3 ),regarding the essential amino acids , the range of ornithine is (1.7-2.3) table 4 ,aspartic acid (1-1.5) (table 5) also this was in accordance to the study done by (Morisietal.,1979) that was carried on 15 selected patients with idiopathic oligozoospermia, 27-39 yr of age.

Control studies were performed on 15 age-matched 
subjects defined as fertile and they found the level of amino acids in fertile men ornithine, Lysine, Histidine, Arginine ,Aspartic acid ,Threonine, Glutamic acid ,Alanine Valine, Isoleucine, Leucine, Tyrosine ,Phenylalanine 0.24, 2.70 ,2.12, 2.79,0.82, 2. 15, 5.35, 0.74, $0.92,1.84,2.45,3.00$, 0.63 respectively while in infertile groups the levels of ornithine, Lysine, Histidine, Arginine Aspartic acid, Threonine, Glutamic acid ,Alanine ,Valine, Isoleucine Leucine ,Tyrosine, Phenylalanine $0.17,0.83,0.86,1.47,0.28,0.93$, $2.110 .22,0.70,1.04,1.01,1.66,0.22$ respectively ,also recent Austrian study about the important role that amino acids have in assisting men with subfertile problems The study assessed changes tosperm quality in sub-fertile males following supplementation with micronutrients. Results revealed that men who took the supplement had a 215 percent improvement in the density of sperm cells, 33 percent improvement in the volume of ejaculated sperm, and a 23 percent improvement in overall sperm motility Arginine, has specific roles associated with male fertility.

Arginine amino acid is found in high concentrations within sperm, particularly in sperm heads. It's also the precursor in the synthesis of spermidine, putrescine and spermine. These three compounds are all important components of sperm. Although sperm may still be produced without adequate levels of arginine, their form is more likely to be abnormal. Poor sperm health will lower fertility. Arginine supplementation can help to boost healthy sperm count and improve fertility.

The body naturally produces L-arginine. However, at times this amino acid can be in short supply. This can be detrimental to reproductive health. Low levels of L-arginine can lead to erectile

dysfunction, low sperm count, decreased sperm motility, and sperm morphological abnormalities.
Elevating L-arginine availability will increase blood flow to the reproductive organs. This makes it easier to develop and maintain erections. Larginine is necessary for the production of nitric oxide,.it also improves nutrient supply to the developing sperm ,supplement with L-arginine will boost sperm count. (Aydin et al., 1995); (Schachter et al., 1973). Forward sperm motility is also improved when regularly taking Larginine supplements - (Morgante et al. ,2010).

\section{Conclusion:}

It is important to measure seminal plasma amino acids in the routine assessment of male fertility.

\section{References:}

Aydin S,Palmer R, AshtonD, MoncadaS.(1995). Vascular endothelial cells synthesize nitric oxide from L-arginine.Research Laboratories, Beckenham, Kent, UK. 333(6174):664-666.

Morgante G,Aniello G, Ronsini S,GuidaF, Spinelli P, D'Aniello A et al.,(2010).Natural ways to enhance male fertility, FertilSteril, (84)5:1444-9.

Morisi G, Malandrino F,Frajese G. (1979). Free Amino Acids in Semen: Measurement and Significance in Normal and Oligozoospermic Men, Arch Androl, 2(3):257-259.

\section{SchachterA,BarbulA,ArnalA,ArmstrongM,Bec kerR,CastilloL et al.,(1973): Orthomolecular medical use of L-citrulline for vasoprotection, relaxative smooth muscle tone and cell protection.Normal Values for Children and Adults", Metab, 22:561-569.}

\section{Silvestroni L,Morisi G, Malandrino F, Frajese} G,(2009). Free Amino Acids in Semen:Measurement and Significance in Normal and Oligozoospermic Men, Arch Androl, 2(3):257261 